\title{
BMJ Open Financial Incentives to Increase Uptake of Pediatric HIV Testing (FIT): study protocol for a randomised controlled trial in Kenya
}

\author{
Anjuli D Wagner, ${ }^{1}$ Irene N Njuguna, ${ }^{2,3}$ Jillian Neary, ${ }^{1}$ Vincent O Omondi, ${ }^{4}$ \\ Verlinda A Otieno, ${ }^{4}$ Joseph Babigumira, ${ }^{1,5}$ Elizabeth Maleche-Obimbo, ${ }^{6}$ \\ Dalton C Wamalwa, ${ }^{6}$ Grace C John-Stewart, ${ }^{1,2,7,8}$ Jennifer A Slyker ${ }^{1,2}$
}

To cite: Wagner AD, Njuguna IN, Neary J, et al. Financial Incentives to Increase Uptake of Pediatric HIV Testing (FIT): study protocol for a randomised controlled trial in Kenya. BMJ Open 2018:8:e024310. doi:10.1136/ bmjopen-2018-024310

- Prepublication history for this paper is available online. To view these files, please visit the journal online (http://dx.doi org/10.1136/bmjopen-2018024310).

Received 21 May 2018 Revised 15 August 2018 Accepted 21 August 2018

Check for updates

(C) Author(s) (or their employer(s)) 2018. Re-use permitted under CC BY-NC. No commercial re-use. See rights and permissions. Published by BMJ.

For numbered affiliations see end of article.

Correspondence to Dr Anjuli D Wagner; anjuliw@uw.edu

\section{ABSTRACT}

Introduction Index case testing (ICT) to identify HIVinfected children is efficient but has suboptimal uptake. Financial incentives (FI) have overcome financial barriers in other populations by offsetting direct and indirect costs. A pilot study found FI to be feasible for motivating paediatric ICT among HIV-infected female caregivers. This randomised trial will determine the effectiveness of $\mathrm{Fl}$ to increase uptake of paediatric ICT.

Methods and analysis The Financial Incentives to Increase Uptake of Pediatric HIV Testing trial is a five-arm, unblinded, randomised controlled trial that determines whether FI increases timely uptake of paediatric ICT. The trial will be conducted in multiple public health facilities in western Kenya. Each HIV-infected adult enrolled in HIV care will be screened for eligibility: primary caregiver to one or more children of unknown HIV status aged 0-12 years. Eligible caregivers will be individually randomised at the time of recruitment in equal 1:1:1:1:1 allocation to one of five arms (US\$0 (control), US\$1.25, US\$2.50, US $\$ 5.00$ and US $\$ 10.00$ ). The trial aims to randomise 800 caregivers. Incentives will be disbursed at the time of child HIV testing using mobile money transfer or cash. Arms will be compared in terms of the proportion of adults who complete testing for at least one child within 2 months of randomisation and time to testing. A cost-effectiveness analysis of FI for paediatric ICT will also be conducted. Ethics and dissemination This study was reviewed and approved by the University of Washington Institutional Review Board and the Kenyatta National Hospital Ethics and Research Committee. Trial results will be disseminated to healthcare workers at study sites, regional and national policymakers, and with patient populations at study sites (regardless of enrolment in the trial). Randomised trials of caregiver-child FI interventions pose unique study design, ethical and operational challenges, detailed here as a resource for future investigations.

Trial registration number NCT03049917; Pre-results.

\section{INTRODUCTION}

Perinatally acquired HIV infection is associated with high morbidity and mortality. ${ }^{1}$ While prompt initiation of antiretroviral therapy
Strengths and limitations of this study

- The five-arm individual-level randomised design with a concurrent control arm will enable a rigorous comparison of paediatric HIV testing uptake between incentivised and unincentivised groups, controlling for background temporal trends.

- The inclusion of four levels of financial incentives (FI) will allow for the direct comparison of uptake between different levels of $\mathrm{FI}$ and identify any asymptotic relationships in the dose-response curve.

- Study staff will aim to randomise all eligible clients very early at the time of first contact to minimise selection bias that is common in randomised trials.

- Randomisation will use a scratch card approach to allow for conceptual transparency in the randomisation process; Fls are disbursed using mobile money transfer technology to reflect the dominant banking practices in a Kenyan setting.

- Trial sites are in western Kenya where paediatric index case testing campaigns have already had high penetration; incentives may have a different effect in settings where such campaigns have been less common.

(ART) reduces mortality and morbidity and promotes growth and development, ${ }^{2-6}$ delayed diagnosis and treatment until a child is severely ill limits the benefits of ART. ${ }^{7-9}$ Global scale-up of prevention of mother-tochild transmission of HIV (PMTCT) systems has markedly reduced the number of new infant HIV infections. ${ }^{10}$ However, many older HIV-infected children remain undiagnosed, either through PMTCT dropout or due to infant infections attributable to incident maternal infection during pregnancy or postpartum $^{11}$ when HIV incidence is high ${ }^{12}$ and repeat maternal HIV testing is low. ${ }^{13}{ }^{14}$ Infant infections due to incident maternal infection are often missed by traditional prevention and early infant diagnosis systems. ${ }^{15}$ 
Index case testing (ICT), where the children of an HIV-infected adult (the 'index case') receiving HIV care are tested for HIV, is an efficient approach to case detection with high prevalence among children tested, but uptake remains suboptimal. ${ }^{16}{ }^{17}$ In a previous study in Nairobi, only $14 \%$ of adults offered systematic paediatric ICT had their child tested for HIV ${ }^{18}$; in this study, barriers to paediatric HIV testing included structural, interpersonal, emotional, logistical and financial issues. ${ }^{19}$ Previous studies have addressed interpersonal and emotional barriers through assisted disclosure and support group interventions, ${ }^{20}$ system-level barriers through medical record flags ${ }^{21}$ and logistical barriers through offering a choice of home-based or clinic-based testing. ${ }^{17}{ }^{18}$ However, these approaches may be expensive and rely on additional healthcare workers. In the context of limited health resources, approaches that minimise costs and maximise uptake are needed. A recent randomised trial showed that small financial incentives (FI) (US\$2) were successful in increasing uptake of HIV testing among children and adolescents aged 8-17 years, using a community-based recruitment approach. ${ }^{22}$ Our team recently completed a feasibility pilot study of small FI to motivate uptake of paediatric ICT, ${ }^{23}$ but there have been no studies to date that have evaluated the effectiveness of FI to increase the uptake of paediatric ICT.

There are several unique ethical, logistical and analytic challenges in designing a study to assess the effectiveness of incentivising caregivers to complete HIV testing for their children. Ethical concerns, including randomising a person of authority to act on another's behalf, assessing child-caregiver relationships to avoid inadvertent disclosure of maternal HIV status, ensuring child's well-being is not compromised and reducing risks of social harms, have been addressed elsewhere. ${ }^{24}$ Logistically and analytically, there are unique challenges in managing FI randomisation and disbursement, minimising the drop-off between recruitment and randomisation, minimising contamination effects leading to presentation of ineligible individuals and accounting for competing interventions in the region.

This study-Financial Incentives to Increase Uptake of Pediatric HIV Testing (FIT) - is a randomised controlled trial (RCT) that determines whether FI increases uptake of paediatric ICT, and determines the cost-effectiveness of various levels of FI. This paper details the study protocol and describes design considerations specific to trials incentivising paediatric testing.

\section{METHODS AND ANALYSIS \\ Conceptual framework}

FI may motivate parents who are willing to test to take action to test by either offsetting costs or by motivating more prompt action. Unwilling parents, who face extreme fear or real dangers from revealing their HIV status, may not be motivated by FI to take action to test. Social services-including enhanced counselling and peer support groups-may help parents move from unwilling to willing. Hypothesis: We hypothesise that the proposed FI intervention will primarily move willing parents from 'Willing to test' to 'Taking action' (figure 1).

\section{Pilot study}

A pilot study (NCT02931422) was conducted between October 2016 and January 2017. In the pilot study $(\mathrm{N}=60)$, values of US $\$ 5$, US $\$ 10$ and US $\$ 15$ were tested; these were based on cost data from a previous paediatric ICT study conducted by the same team in Nairobi. ${ }^{18}$ The lowest incentive value reflected the 75th percentile of direct non-medical costs (transportation, childcare and food/ drink outside the home), the middle value reflected the 75th percentile of direct non-medical and indirect costs (lost wages from paid and unpaid work), and the highest value reflected the direct costs, indirect costs and a second day of lost wages. ${ }^{23}$

\section{Study design}

The FIT trial is a five-arm, unblinded, individual-level, superiority RCT of FI. Eligible individuals will be randomised using a 1:1:1:1:1 allocation to no incentive, US $\$ 1.25$, US $\$ 2.50$, US $\$ 5.00$ or US $\$ 10.00$ (2016 US $\$$ ) (figure 2). Randomised individuals can redeem the value of their incentive upon completing testing with study staff within 2 months of randomisation. The study will employ a roving, multisite model in which multiple clinic sites run concurrently, but each site will only be active for recruitment for 2 months. This model was selected to limit the extent to which clients at the facilities became aware of the FI opportunity through word of mouth, to limit the number of clients screened more than once (as most clients visit the clinic every 3 months) and to allow for sampling approximately proportional to facility size.

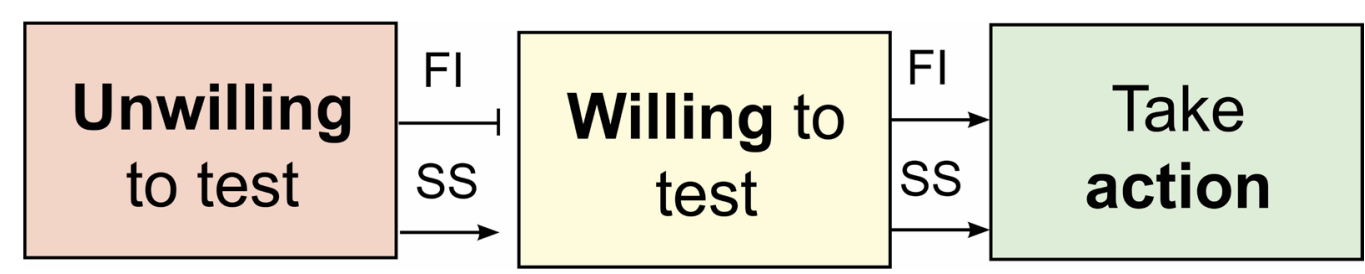

Figure 1 Conceptual framework. Financial incentives (FI) may motivate caregivers who are willing to test to move to take action to test. However, they are unlikely to motivate caregivers who are unwilling to test to take action. Social services (SS) interventions may be needed to move those parents who are unwilling to test to take action. 


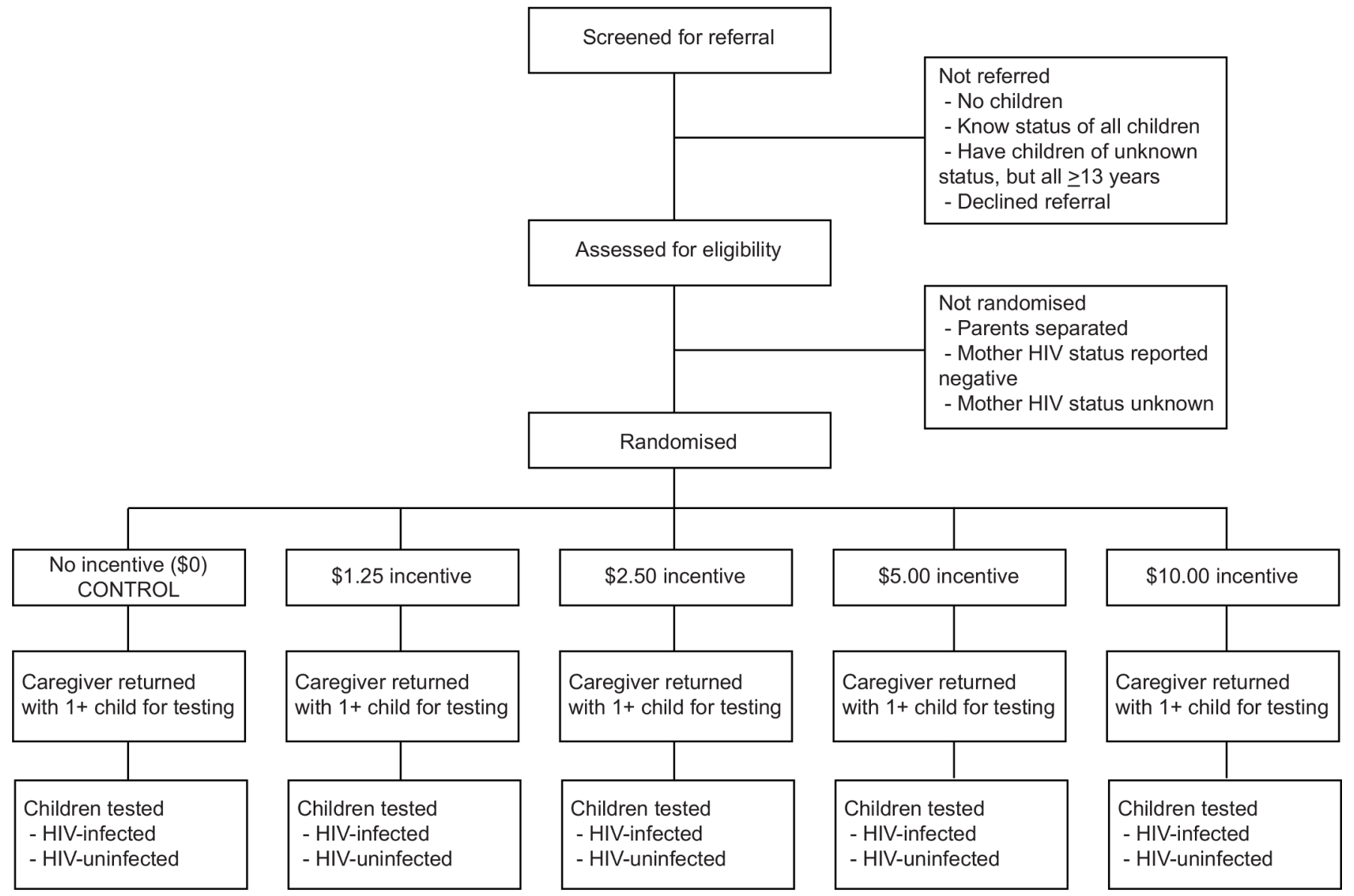

Figure 2 Consolidated Standards of Reporting Trials (CONSORT) diagram.

\section{Design considerations}

Alternative study designs that included historic and lead-in control periods, as well as cluster randomisation were considered, but ultimately not selected. Historic and lead-in control periods from within the same study sites could have suffered from depletion of susceptibles, in which the virtually fixed target population of index cases decreases over time as the most susceptible individuals experience the outcome of interest (eg, complete ICT for their children). While new individuals are diagnosed with HIV each day, the rate at which those individuals are added to the population in care is slow relative to the number of individuals active in care at the beginning of a study. Additionally, there are many events-rapid HIV testing campaigns, school holidays, guideline changes-that could have occurred during either the control or intervention periods and led to temporal trends that could not be robustly controlled for. A concurrent control arm was considered to limit the extent to which these temporal and epidemiologic trends would impact the estimation of the effect of FI on testing.

A cluster RCT (cRCT) was also considered, which would have limited contamination; in the context of this study, contamination would have been the extent to which individuals within a clinic became aware of the other values of FI being offered and discouraged by receiving less than the maximum FI value. However, a cRCT design for a five-arm trial would have required a prohibitively large number of clinics to detect meaningful differences in uptake, which was not feasible.

\section{Determination of incentive values}

Trial incentive values

Incentive values were determined using results from the FIT pilot study described above. ${ }^{23}$ Uptake of testing in the pilot study was high and comparable between the three arms $\left(75 \%, 70 \%\right.$ and $75 \%$ across arms, respectively). ${ }^{23}$ Because it was unclear whether uptake was similar across the pilot study FI values because we had reached the top of the demand curve (eg, where even higher FI would yield no increase in testing) or whether we were clustered in the middle of a demand curve (where higher or lower values would provide further differences in testing uptake), we decided to widen the range of FI values to remove the highest value and include lower values.

The trial incentive values will be US $\$ 1.25$, US $\$ 2.50$, US $\$ 5.00$ and US $\$ 10.00$, and a control with no FI (US\$0). Participants will be compensated and additional US $\$ 3.00$ for transportation costs regardless of arm; this reimbursement will be included to ensure more equitable benefit for those in the control arm for research participation, and will not be described to participants prior to the testing visit in order to not act as an additional incentive. 


\section{Incentive considerations}

Alternative formats of incentives were considered, including lottery-based incentives and non-FIs such as household items or agricultural items. Lottery-based incentives were considered less acceptable by some co-investigators due to a perception that this was similar to gambling, which has a negative connotation for some religious groups in Kenya. Agricultural and household items (and non-financial commodities in general) were felt to be more attractive for those living in rural settings, but had additional costs involved in procurement, distribution and tracking of commodities, which would increase programme costs. Cash or mobile money transfer was thus adopted as the most fungible, widely acceptable, accountable and low-cost FI format to deliver in the context of an intervention.

\section{Patient and public involvement}

The research intervention and outcome were informed by formative research with the patient population: the concept of FI emerged from qualitative and quantitative work with patients ${ }^{19}$; the value and format of the FI were reviewed by patients during the pilot. ${ }^{23}$ Site-specific, and overall, study results will be shared with the research facilities in close-out meetings; we do not have ethical permission to recontact individual study participants to share study results.

\section{Study sites}

The trial will be conducted at several government health facilities in western Kenya, including facilities in Kisumu, Siaya and Homa Bay counties. Study team members will simultaneously operate up to three sites, and then relocate to new sites following 2 months of recruitment. Facilities will be selected 1-2 months in advance and approved by the county health director's office and facility heads. Sites will be selected based on high volume of adults in HIV care and relatively low penetration of recent paediatric ICT campaigns or programmes. A full list of study sites will be provided in the trial results manuscript.

\section{Recruitment processes and eligibility criteria}

Index adult clients attending the HIV care clinics will be screened by study staff to determine eligibility: being HIV infected and having one or more children of unknown HIV status aged 0-12 years. Children will be considered of unknown status if they have never been tested for HIV or tested negative during infancy but did not complete confirmatory negative testing after 18 months or following cessation of breastfeeding. Index client caregivers will be allowed to test any child formally in their care, both biological children and children to whom they serve as guardian. This decision was made to address the high burden of undiagnosed HIV infection among orphans and vulnerable children, and the ethical obligation to include them in potentially beneficial interventions. There are no restrictions regarding concomitant care or interventions for caregiver participation.
For male index cases, an additional eligibility criterion is that the child's mother is HIV infected. For male clients who do not know the status of the child's mother, the index will not be randomised until maternal testing has been offered. Male clients with children whose biological mother has died are eligible.

\section{Design considerations}

Recruitment staff will aim to screen every client who passes through the clinic to accurately measure the true absolute and relative denominator of eligible adults. All approached clients will be invited to provide oral informed consent for eligibility determination and randomisation. Eligibility (number of children and child HIV test history) will be assessed at recruitment, before potential participants are informed about the incentives, in order to reduce the likelihood of caregivers bringing in children of known HIV status or children who are not their own. No instances of inappropriate testing or deception were uncovered in the pilot study.

\section{Randomisation}

Caregivers will be randomised immediately following determination of eligibility in order to minimise bias associated with the attrition between referred and enrolled participants, which is common in RCTs. Caregivers will be invited to select a scratch card from an opaque bag and to scratch the metallic strip to reveal their randomisation arm (figure 3). This randomisation allocation technique has been used previously in this setting. ${ }^{25}$ Minimal optional data will be collected at the time of randomisation, which link scratch cards from origin to testing, including caregiver age and sex, number of children and telephone contact number. Caregivers will be invited, but not required, to provide these data at the time of randomisation; data will be collected to link caregivers to their randomisation values in case the scratch card is lost. Caregivers will be called up to three times after randomisation to schedule/reschedule enrolment and testing visits.

Randomisation sequence was generated by a statistician not involved in the study using STATA V.14 using the 'ralloc' command. Scratch cards were manufactured by Scratch Off Systems (www.scratchoff.com); 800 cards were created in batches of 15 (three cards with each of the five randomisation arms per batch). Fixed size batches were selected instead of variable size batches to allow for batches to be fully used at each facility site to ensure relative balance of the five study arms within a site, and therefore balance of the sites between the five arms.

Blinding: Caregivers and study clinic staff will not be blinded to participant allocation after randomisation, given the nature of the incentive allocation. Study staff who are not directly involved in participant management (including study coordinators, data managers and analysts, and other coinvestigators) will be blinded to participant study arm. Unblinding will be permissible in the event of adverse event reporting; participant 

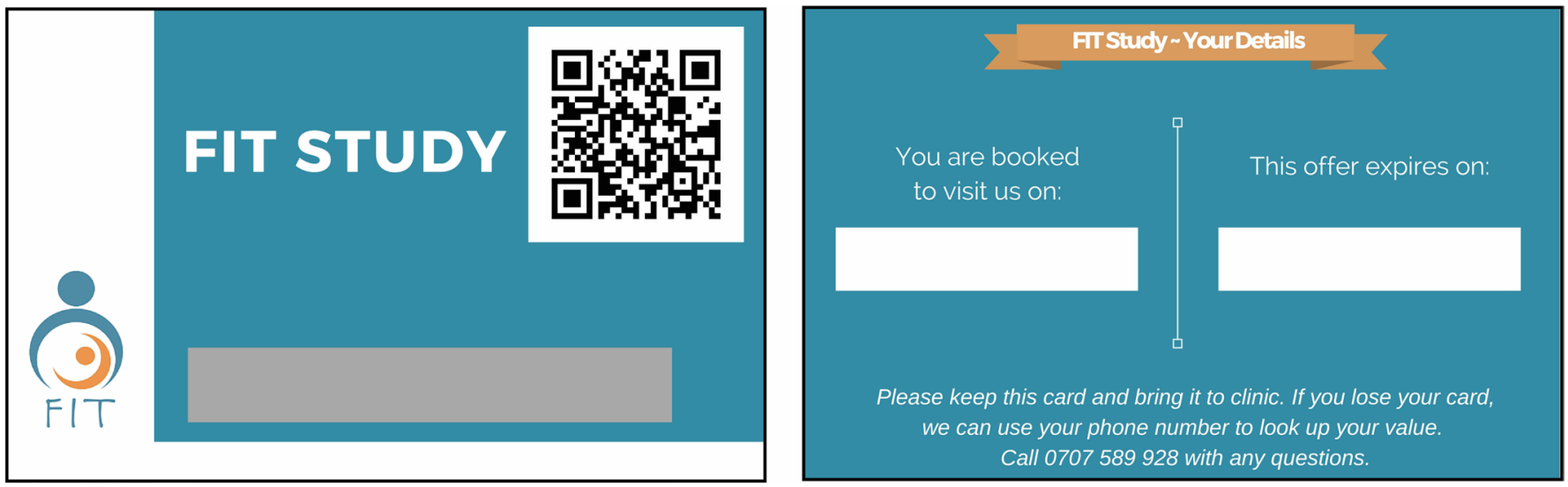

Figure 3 Randomisation scratch card before randomisation arm reveal. FIT, Financial Incentives to Increase Uptake of Pediatric HIV Testing.

allocation arm will be requested from the statistician who conducted the randomisation.

\section{Enrolment and testing}

Index participant enrolment

Enrolment and child testing will occur during the same visit, usually after randomisation, although same-day enrolment and testing will be allowed. Caregivers will provide written consent for child testing, and given the option of having their older children ( $\geq 7$ years) provide assent for study participation. Enrolment and testing visits must occur within 2 months of randomisation in order to receive the $\mathrm{FI}$; individual exceptions will be made to accommodate school and national holidays. Testing during weekdays and weekends is allowed. At the time of enrolment, detailed information about caregiver demographics, testing and treatment history, income and costs, and child's PMTCT, health and testing history will be collected. Participants will be also screened to determine whether they are at risk of intimate partner violence and referred to existing clinical services as appropriate.

\section{Child testing services}

Children will be tested according to the Kenyan National HIV Testing Guidelines. ${ }^{26}$ At the time of protocol development, children $>18$ months are tested by rapid HIV test kit; those who test positive during the first test kit are tested by a second rapid test kit; discrepant results are referred to an HIV care clinic for repeat of the rapid test algorithm. HIV-exposed children 0-18 months are tested using DNA PCR on a filter paper. Children diagnosed as HIV positive will be referred to the HIV care clinic of the caregiver's choice. Children who are identified as having ongoing HIV exposure (eg, breastfeeding or lacking final confirmatory test 6 weeks after cessation of breastfeeding) will be referred to the PMTCT clinic of the caregiver's choice for continued prophylaxis and infant testing (table 1).

\section{Cash disbursement and accounting}

FI values and travel reimbursement will be disbursed to caregivers at the end of the testing visit. Caregivers will be given the choice of receiving the incentive using mobile money transfer (transfer fees paid by the study) or in cash. Each financial transfer (cash or mobile money) will be recorded in an accounting log, and the randomisation card will be collected.

\section{Participant follow-up}

Caregivers and their children who test HIV negative will not be followed beyond the testing visit. Caregivers with one or more children who test HIV positive will be contacted by phone or clinic visit at 1, 3, 6, 9 and 12 months after diagnosis, or until linkage to care has been determined, to assess linkage to care, child treatment status,

Table 1 Adapted Standard Protocol Items:

Recommendations for Interventional Trials (SPIRIT) diagram

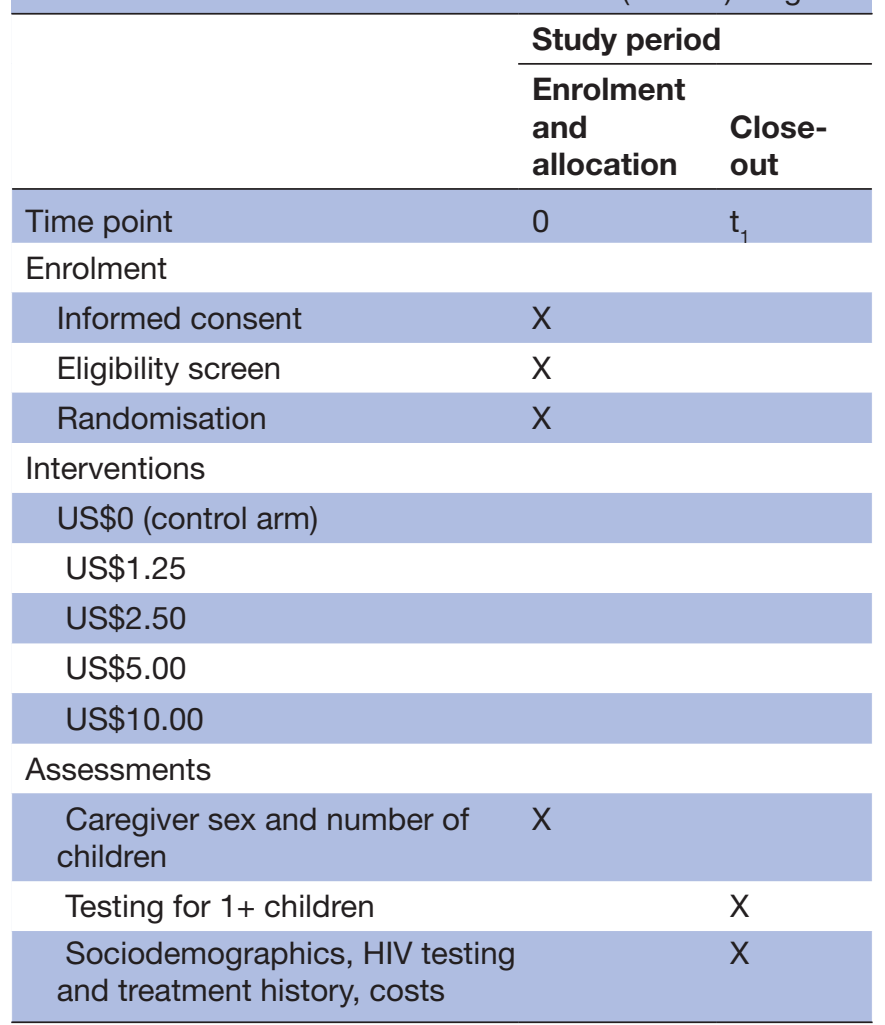


emotional coping, risk of violence and child welfare. Any social harms noted during this follow-up will be referred or reported, as appropriate, using existing systems within the Kenyan public health and legal systems.

\section{Discontinuation, withdrawal or allocation modification}

Participants may withdraw consent for participation at any point after randomisation; principal investigators may withdraw a participant from the study on a caseby-case basis if the study intervention poses a risk to the participant. Participants who withdraw consent for participation will not be contacted further by the study team. Participants who are randomised but do not complete testing within the 2-month window will be considered as non-testers and included in the final analysis.

\section{Data collection and management}

Study staff will use mobile phones and tablets to collect data. Electronic data collection improves data accuracy by eliminating the extra step of entering data from paper forms into an electronic database. ${ }^{27}$ The programme used to collect and store the data is entitled Open Data Kit and is available as an open-source platform. ${ }^{28}$ Data will be stored on the password-protected phone/tablet until they are uploaded through an encrypted connection to the study's secure electronic database, at which point they will be automatically deleted from the phones and no longer accessible. Weekly enrolment and testing reports will be generated to track study progress and ensure quality data collection. Study investigators will have access to the deidentified, unblinded data set after follow-up is completed.

\section{Outcome measures}

The primary study outcomes are: (1) proportion of index cases who complete paediatric HIV testing for one or more children within 2 months of randomisation, and (2) time to HIV testing completion. A priori stratified analyses will be conducted, stratified by caregiver sex, caregiver age and whether the caregiver has one or more than one eligible child for testing.

\section{Sample size and power analysis}

Eight hundred index cases will be randomised; given 160 adults in each of five randomisation arms, we will have $>80 \%$ power to detect a minimum of $10 \%-20 \%$ difference in uptake between each of the arms (table 2). Assumptions about uptake for the unincentivised group were based on data from the previously conducted unincentivised testing study in the same population. ${ }^{18}$ We will have sufficient power to detect differences over a range of uptake scenarios (table 2). All power calculations are shown using pairwise comparisons between randomisation arms (eg, US $\$ 0$ vs US\$1.25); we will have additional power for comparisons between arms with larger separation of FI values (eg, US $\$ 1.25$ vs US $\$ 10.00$ ).

\section{Statistical methods and analysis \\ Primary outcome analyses}

We will compare the proportion of index cases bringing at least one child for testing within 2 months between groups randomised to control versus each of the four FI levels using a generalised linear model (GLM) with log link and binomial or Poisson distribution, adjusting for facility. If randomisation fails to balance potential confounders, we will perform the aforementioned analysis, adjusting for unbalanced confounders. We will additionally compare the time to testing between each of the five arms using a stratified Cox proportional hazards regression model, which adjusts for facility in estimating a pooled HR, and adjusting for unbalanced confounders as necessary. Primary outcome analyses will include a Hochberg's adjustment to $\mathrm{p}$ values to address multiplicity. We will conduct intent-to-treat analyses as the primary analyses. We will conduct a modified intent-to-treat analysis (removing any individuals randomised but found to be ineligible following randomisation) as a sensitivity analysis. Multiple imputation will be conducted to address any data missingness in outcomes or confounders. Complete case analysis will be conducted as a sensitivity analysis.

\section{Stratified analysis}

Both primary outcome analyses will additionally be performed stratified by caregiver sex, caregiver age (above and below median age) and number of eligible children (dichotomised as one child or more than one child). These analyses will include a Hochberg's adjustment to $\mathrm{p}$ values to address multiplicity.

\section{Secondary analyses}

Characteristics of testers between arms

We will compare index-level and child-level characteristics between testers in each arm. We will compare the following index case characteristics between index cases who completed testing in each arm: income, sex, partnership status, history of HIV testing and treatment, and number of eligible children in the house. We will

Table 2 Power calculations

\begin{tabular}{lllllllll}
$\begin{array}{l}\text { No } \\
\text { incentive (\%) }\end{array}$ & Power (\%) & US\$1.25 (\%) & Power (\%) & US\$2.50 (\%) & Power (\%) & US\$5.00 (\%) & Power (\%) & US\$10.00 (\%) \\
\hline 13 & $>99$ & 40 & 95 & 60 & 98 & 80 & 71 & 90 \\
13 & 96 & 30 & 96 & 50 & 96 & 70 & 90 & 85 \\
13 & 84 & 26 & 82 & 40 & 95 & 60 & 82 & 75 \\
13 & 39 & 20 & 54 & 30 & 96 & 50 & 78 & 65 \\
\hline
\end{tabular}


use GLM and control for facility. We will compare the following characteristics between children who were tested in each arm: sex, history of HIV testing, number of eligible children in the house and sibling HIV status. We will use generalised linear mixed models, clustering on index case and controlling for facility.

\section{Differences in uptake based on proportion of eligible clients}

We will test the association between the proportion of clients eligible for randomisation in a facility and uptake of testing across incentive values. We hypothesise that sites with fewer eligible clients will have lower levels of uptake across incentive values than sites with a greater proportion of eligible clients. This is hypothesised because sites with a lower proportion of eligible index cases have likely already benefitted from interventions to motivate those individuals who are 'willing to test' to 'take action' (figure 1), leaving a disproportionate number of index cases who are 'unwilling to test,' a population that is less susceptible to an FI intervention.

\section{ETHICS AND DISSEMINATION}

\section{Ethical considerations}

FIs, while commonly used to motivate various health behaviours, also commonly raise ethical concerns related to coercion, undue inducement and lack of voluntariness. The study team engaged in discussions with Kenyan paediatricians and other healthcare workers, Kenyan programme implementers and with Treuman Katz Center for Pediatric Bioethics (Seattle Children's Hospital) in the USA. The bioethics consultation offered several clarifying points, which are described elsewhere. ${ }^{24}$

The study was first posted on ClinicalTrials.gov (NCT03049917) on 10 February 2017. This slight delay occurred as we initially attempted to register both the pilot and trial phases of the study as two protocols under one record, but this was ultimately deemed infeasible and confusing, so the trial phase was registered as a separate record. A data monitoring committee will not be convened due to no planned interim analyses and minimal risk potential of the intervention. A steering/ management committee was not deemed applicable in this trial.

\section{Trial status}

This trial began recruitment and enrolment on 31 January 2017. It is anticipated to close recruitment in July 2018 and enrolment in September 2018.

\section{Dissemination plans}

We will plan to share trial results with healthcare workers at study sites, regional and national policymakers and with patient populations at study sites (regardless of enrolment in the trial). We have deposited the full protocol on a publicly available website through the National Clinical Trials registry. We will use the International Committee of
Medical Journal Editors criteria for authorship and will not hire professional writers.

\section{DISCUSSION}

FIs have been effective to promote a variety of desired health behaviours, including adult and adolescent HIV testing. It is important to assess whether this intervention is effective to promote timely uptake of paediatric ICT in sub-Saharan African settings where HIV-related morbidity and mortality remain high for undiagnosed children. This study is the first that we are aware of to test FI to improve uptake of ICT for children.

The pilot study conducted by this team evaluated three levels of FI and saw high, but flat, uptake of testing across the study arms. ${ }^{23}$ Therefore, the larger trial will evaluate a wider and lower range of FI, including a non-incentivised control arm. This five-arm RCT with a concurrent control arm will enable inference about the effect of FIs generally, and at various levels, to promote timely uptake of paediatric ICT.

This study has faced several operational challenges to date. The Kenyan public health system has faced two large nationwide healthcare worker strikes (doctors' strike from December 2016 to February 2017; nurses' strike from May to November 2017), which limited the number of patients presenting for services. Additionally, there was a contested presidential election in August 2017 and a contested repeat election in October 2017, which produced widespread disruption of service provision. To overcome these challenges, the study increased the number of sites enrolling concurrently to achieve the desired sample size.

\section{Study limitations}

The study sites represent one geographical region in Kenya, which may not be generalisable to other settings with lower HIV prevalence or different social dynamics, including HIV-related stigma. Clinics will be chosen to maximise the number of clients enrolled, and therefore will represent mostly high-volume sites; volume of clinic is not expected to influence uptake of testing, but any bias that might occur as a result of clinic selection would likely apply equally to all five randomisation arms, influencing absolute but not relative estimates of uptake. Kenya has had widespread ICT campaigns nationwide in the past 4 years, leaving relatively few individuals in care with undiagnosed children in their care; the impact of FI might be expected to differ in a population of HIV-infected caregivers who were ICT naive. This trial does not include qualitative work to investigate the mechanism of FI. Finally, this intervention targets children aged 0-12 years for testing; however, many HIV-infected adults report having adolescent children (age $\geq 13$ ) of unknown HIV status at home, ${ }^{23}$ who were not eligible for the current trial. Alternative strategies to target and provide acceptable and accessible HIV testing services to adolescents are critically needed. 


\section{CONCLUSION}

In summary, this large multisite RCT will produce robust data on the effect and cost-effectiveness of varying levels of FI on uptake of paediatric ICT. Additionally, this paper describes design considerations and lessons learnt that can be broadly informative in the design of paediatric HIV trials as well as the design of FI trials.

\section{Author affiliations \\ ${ }^{1}$ Department of Global Health, University of Washington, Seattle, Washington, USA 2Department of Epidemiology, University of Washington, Seattle, Washington, USA \\ ${ }^{3}$ Research and Programs, Kenyatta National Hospital, Nairobi, Kenya \\ ${ }^{4}$ Kenya Pediatric Research Consortium, Kenya Pediatric Association, Nairobi, Kenya \\ ${ }^{5}$ School of Pharmacy, University of Washington, Seattle, Washington, USA \\ ${ }^{6}$ Department of Pediatrics and Child Health, University of Nairobi, Nairobi, Kenya \\ ${ }^{7}$ School of Medicine, University of Washington, Seattle, Washington, USA \\ ${ }^{8}$ Department of Pediatrics, University of Washington, Seattle, Washington, USA}

Acknowledgements We would like to acknowledge the FIT Study Clinic Staff (Pamela Agola, Lukio Agalo, Anne Misumi, Joseph Orondo), the Kisumu and Siaya County Directors of Health, and all the facility staff, the biostatistics team (Ken Tapia, Joshua Stern, Barbra Richardson) in Seattle and the UW-Kenya operations staff, Kenya Pediatric Research Consortium (KEPRECON) in Nairobi and Collaborative Initiative for Pediatric HIV Education and Research (CIPHER) staff for their support on this project. We thank the Kizazi Working Group and University of Washington Global Center for the Integrated Health of Women, Adolescents, and Children, (Global WACh) for comments and insights provided during study design and manuscript development. Most of all, we thank the families who have and will participate in the study.

Contributors INN and JAS are the principal investigators and supervised the trial protocol development and implementation. ADW, INN, JN, JB, EMO, DCW, GCJS and JAS participated in designing the trial and data collection tools. VOO and VAO coordinated the study and collected study data. ADW, INN, GCJS and JAS are responsible for the statistical design of the trial and data analysis. ADW, INN and JAS wrote the first draft of the manuscript. All authors critically revised, read and approved the final manuscript.

Funding This work was sponsored by a Center for AIDS Research (CFAR) International Pilot Award, which is supported through the National Institutes of Health (NIH) award P30AI027757, and by the Collaborative Initiative for Pediatric HIV Education and Research (CIPHER) International AIDS Society (323-NJU-TRIAL). Additional support was provided by the UW Global Center for Integrated Health of Women, Adolescents and Children (Global WACh) and the University of Washington CFAR (P30 Al027757). This publication was supported in part by the National Institute of Child Health and Development (NICHD) F32HD088204 to ADW and the Fogarty International Center (FIC) R25 TW009345 and D43TW009783 to ADW and INN, respectively.

Disclaimer The contents of the study are solely the responsibility of the authors and do not necessarily represent the official views of the sponsors. The sponsors gave feedback to the study investigators during the trial design phase, but did not make the ultimate decision about study design. The sponsors do not have any role in the study data collection, management, analysis and interpretation, writing of reports, nor decisions to submit reports for publication.

Competing interests None declared.

Patient consent Not required.

Ethics approval University of Washington Institutional Review Board (UW IRB) and Kenyatta National Hospital Ethics and Research Committee (KNH ERC).

Provenance and peer review Not commissioned; externally peer reviewed.

Open access This is an open access article distributed in accordance with the Creative Commons Attribution Non Commercial (CC BY-NC 4.0) license, which permits others to distribute, remix, adapt, build upon this work non-commercially, and license their derivative works on different terms, provided the original work is properly cited, appropriate credit is given, any changes made indicated, and the use is non-commercial. See: http:// creativecommons.org/licenses/by-nc/4.0/.

\section{REFERENCES}

1. Newell ML, Coovadia H, Cortina-Borja M, et al. Mortality of infected and uninfected infants born to HIV-infected mothers in Africa: a pooled analysis. Lancet 2004;364:1236-43.

2. Cotton MF, Rabie H. Impact of earlier combination antiretroviral therapy on outcomes in children. Curr Opin HIV AIDS 2015;10:12-17.

3. Cotton MF, Violari A, Otwombe K, et al. Early time-limited antiretroviral therapy versus deferred therapy in South African infants infected with HIV: results from the children with HIV early antiretroviral (CHER) randomised trial. Lancet 2013;382:1555-63.

4. Violari A, Cotton MF, Gibb DM, et al. Early antiretroviral therapy and mortality among HIV-infected infants. N Engl J Med 2008;359:2233-44.

5. McGrath CJ, Chung MH, Richardson BA, et al. Younger age at HAART initiation is associated with more rapid growth reconstitution. AIDS 2011;25:345-55.

6. Benki-Nugent S, Eshelman C, Wamalwa D, et al. Correlates of age at attainment of developmental milestones in HIV-infected infants receiving early antiretroviral therapy. Pediatr Infect Dis $J$ 2015;34:55-61.

7. Njuguna IN, Cranmer LM, Otieno VO, et al. Urgent versus poststabilisation antiretroviral treatment in hospitalised HIV-infected children in Kenya (PUSH): a randomised controlled trial. Lancet HIV 2018;5.

8. Wamalwa D, Lehman DA, Benki-Nugent S, et al. Long-term virologic response and genotypic resistance mutations in HIV-1 infected Kenyan children on combination antiretroviral therapy. J Acquir Immune Defic Syndr 2012.

9. Wagner A, Slyker J, Langat A, et al. High mortality in HIV-infected children diagnosed in hospital underscores need for faster diagnostic turnaround time in prevention of mother-to-child transmission of HIV (PMTCT) programs. BMC Pediatr 2015;15:10.

10. UNAIDS. UNAIDS Data 2017. 2017.

11. Johnson LF, Stinson K, Newell ML, et al. The contribution of maternal HIV seroconversion during late pregnancy and breastfeeding to mother-to-child transmission of HIV. J Acquir Immune Defic Syndr 2012;59:417-25.

12. Drake AL, Wagner A, Richardson B, et al. Incident HIV during pregnancy and postpartum and risk of mother-to-child HIV transmission: a systematic review and meta-analysis. PLoS Med 2014;11:e1001608.

13. Rogers AJ, Weke E, Kwena Z, et al. Implementation of repeat HIV testing during pregnancy in Kenya: a qualitative study. BMC Pregnancy Childbirth 2016;16:151.

14. Rogers AJ, Akama E, Weke E, et al. Implementation of repeat HIV testing during pregnancy in southwestern Kenya: progress and missed opportunities. J Int AIDS Soc 2017;20:e25036.

15. Njuguna IN, Wagner AD, Cranmer LM, et al. Hospitalized children reveal health systems gaps in the mother-child HIV care cascade in Kenya. AIDS Patient Care STDS 2016;30:119-24.

16. Govindasamy D, Ferrand RA, Wilmore SM, et al. Uptake and yield of HIV testing and counselling among children and adolescents in subSaharan Africa: a systematic review. J Int AIDS Soc 2015;18:20182.

17. Ahmed S, Sabelli RA, Simon K, et al. Index case finding facilitates identification and linkage to care of children and young persons living with HIV/AIDS in Malawi. Trop Med Int Health 2017;22:1021-9.

18. Wagner AD, Wachira CM, Njuguna IN, et al. Active referral of children of HIV-positive adults reveals high prevalence of undiagnosed HIV. $J$ Acquir Immune Defic Syndr 2016.

19. Wagner AD, O’Malley G, Firdawsi O, et al. Brief report: disclosure, consent, opportunity costs, and inaccurate risk assessment deter pediatric hiv testing: a mixed-methods study. J Acquir Immune Defic Syndr 2018;77:393-9.

20. Lewis Kulzer J, Penner JA, Marima R, et al. Family model of HIV care and treatment: a retrospective study in Kenya. $J$ Int AIDS Soc 2012;15:8.

21. Mongare JOF, Ojoo S, Ooko H, et al. Client-centered HIV testing and counseling as a strategy for scaling up access to HIV prevention and care services. 7th IAS Conference on HIV Pathogenesis. Kuala Lumpur, Malaysia, 2013.

22. Kranzer K, Simms V, Bandason T, et al. Economic incentives for HIV testing by adolescents in Zimbabwe: a randomised controlled trial. Lancet HIV 2018;5:e79-86.

23. Njuguna IN, Wagner AD, Omondi VO, et al. Financial incentives for pediatric HIV testing in Kenya. Pediatr Infect Dis J 2018:1.

24. Wagner AD, Shah SK, Njuguna IN, et al. Letter to the Editor: financial incentives to motivate pediatric HIV testing - assessing the potential for coercion, inducement, and voluntariness. J Acquir Immune Defic Syndr 2018.

25. Yotebieng M, Thirumurthy $\mathrm{H}$, Moracco KE, et al. Conditional cash transfers to increase retention in PMTCT care, antiretroviral 
adherence, and postpartum virological suppression: a randomized controlled trial. J Acquir Immune Defic Syndr 2016;72(Suppl 2):S124-9.

26. Ministry of Health. Guidelines on use of antiretroviral drugs for treating and preventing HIV infection in Kenya. Nairobi, Kenya: NASCOP, 2016.
27. Byass $\mathrm{P}$, Hounton $\mathrm{S}$, Ouédraogo M, et al. Direct data capture using hand-held computers in rural Burkina Faso: experiences, benefits and lessons learnt. Trop Med Int Health 2008;13(Suppl 1):25-30

28. ICTD. Open data kit: tools to build information services for developing regions [program]. 1 version. 2010. 